\title{
Association of primary immune thrombocytopenia and common allergic diseases among children
}

\author{
Ming-Ru Chiang ${ }^{1}$, Chang-Ching Wei ${ }^{2,3}$, Chin-Shin Muo ${ }^{4}$, Lin-Shien Fu' ${ }^{1}$ Tsai-Chung Li ${ }^{5}$ and Chia-Hung Kao ${ }^{6,7}$
}

\begin{abstract}
BACKGROUND: Growing evidence has revealed a link between autoimmune and allergic diseases. However, few studies have assessed the relationship between allergic diseases and primary immune thrombocytopenia (ITP), an autoimmune disease frequently occurring in children. This population-based case-control study investigated the association between common allergic diseases and the subsequent risk of developing ITP during childhood.
\end{abstract}

METHODS: This study investigated 1,203 children younger than 18 y of age who were diagnosed with ITP between 1998 and 2008, as well as 4,812 frequency-matched controls. The odds ratios of the association between ITP and preexisting allergic diseases were calculated.

RESULTS: Children with every type of allergic disease examined in this study (except asthma) exhibited an increased risk of developing ITP; the lowest adjusted odds ratio (aOR) was 1.39 for allergic conjunctivitis (95\% confidence interval $(\mathrm{Cl})=1.09$ 1.79), whereas the greatest aOR was 1.84 for allergic rhinitis (95\% Cl $=1.49-2.27)$. The aORs increased with the number of concurrent allergic diseases to $2.89(95 \% \mathrm{Cl}=1.98-4.22)$ for children with at least three allergic diseases.

CONCLUSION: Children with atopic diathesis have a greater risk of subsequently developing ITP. The fundamental determinants of this relationship warrant further study.

$\mathbf{P}$ rimary immune thrombocytopenia (ITP) is an acquired autoimmune disease characterized by isolated thrombocytopenia with normal bone marrow and the absence of other causes of thrombocytopenia $(1,2)$. Antiplatelet antibodies secreted by autoreactive B lymphocytes resulting in platelet destruction through the reticuloendothelial system were described as the primary immunological defect in ITP. ITP was reported as a type-1 T-cell (Th1)-predominant disease, and activated platelet-specific autoreactive $\mathrm{T}$ cells that drive the generation of platelet reactive autoantibodies by $\mathrm{B}$ cells are present in ITP. In contrast to ITP, allergic disorders are considered type-2 T-cell (Th2)-related immune responses with chronic inflammation at sites of persistent or repetitive exposure to allergens. Allergic conjunctivitis $(\mathrm{AC})$, allergic rhinitis (AR), atopic dermatitis (AD), asthma, and urticaria are frequently observed organ-specific allergic diseases involving IgE-mediated inflammation, and increasing epidemiological evidence has indicated a potential link between Th2-related allergic diseases and Th1-related autoimmune diseases (3-5). However, the relationship between pre-existing allergic diseases and ITP risk remains unclear. Therefore, in this nationwide population-based case-control study, we investigated the relationship between common allergic diseases and subsequent risk of developing ITP in children.

\section{RESULTS}

We selected 1,203 children younger than $18 \mathrm{y}$ of age with ITP diagnosis as well as 4,812 controls. The proportion of girls and boys was nearly equal ( 49.5 vs. $50.5 \%$ ), and the average age was 6.18 y $(S D=5.20)$ in the ITP group. No significant differences were observed in the urbanization of residential area between the ITP children and controls. However, a history of allergies, including AR (17.2 vs. $8.96 \%)$, AC (8.98 vs. $5.36 \%)$, asthma ( 8.65 vs. $5.24 \%), \mathrm{AD}(6.98$ vs. $3.66 \%)$, and urticaria (4.16 vs. $2.41 \%$ ), was more common in the ITP children than in the controls (Table 1).

The children with AR exhibited the highest risk of developing ITP $(\mathrm{OR}=1.84 ; 95 \% \mathrm{CI}=1.49-2.27)$, followed by those with $\mathrm{AD}(\mathrm{OR}=1.66 ; 95 \% \mathrm{CI}=1.25-2.21)$ and $\mathrm{AC}(\mathrm{OR}=1.39$; $95 \%$ CI $=1.09-1.79)$ (Table 2). Compared with the children with no allergic disease, the risk increased with the number of allergic diseases from 1.94 to $2.89(P<0.0001)$. Both sexes exhibited the same trend, except for boys with AC.

Table 3 shows the association between allergic disease and ITP among the age groups. The children with AR exhibited a higher risk of developing ITP at ages younger than $2 \mathrm{y}$ compared with children of the same age without AR. The children aged 1-2 and 6-11 y have a higher risk of developing AD. The 6-11-y-old children with urticaria had a $22 \%$ increased odds for ITP compared with the corresponding age group of controls without urticaria $(95 \% \mathrm{CI}=1.22-4.01)$.

\footnotetext{
The first two authors contributed equally to this work.

'Department of Pediatrics, Taichung Veterans General Hospital, Taichung, Taiwan; '2Department of Nephrology, Children's Hospital, China Medical University Hospital, Taichung, Taiwan; ${ }^{3}$ College of Medicine, China Medical University, Taichung, Taiwan; ${ }^{4}$ Management Office for Health Data, China Medical University Hospital, Taichung, Taiwan; ${ }^{5}$ Department of Public Health, China Medical University, Taichung, Taiwan; ${ }^{6}$ Department of Nuclear Medicine and PET Center, China Medical University Hospital, Taichung, Taiwan; ${ }^{7}$ Graduate Institute of Clinical Medical Science and School of Medicine, College of Medicine, China Medical University, Taichung, Taiwan. Correspondence: Chia-Hung Kao (d10040@mail.cmuh.org.tw)
} 
Table 1. Demographics between primary immune thrombocytopenia (ITP) and non-ITP controls

\begin{tabular}{|c|c|c|c|c|c|}
\hline \multirow[b]{2}{*}{ Variable } & \multicolumn{2}{|c|}{$\begin{array}{c}\text { ITP; } \\
N=1,203\end{array}$} & \multicolumn{2}{|c|}{$\begin{array}{l}\text { Non-ITP; } \\
N=4,812\end{array}$} & \multirow[b]{2}{*}{$P$ value } \\
\hline & $n$ & $\%$ & $n$ & $\%$ & \\
\hline \multicolumn{6}{|l|}{ Age, year } \\
\hline $1-2$ & 439 & 36.5 & 1,756 & 36.5 & \\
\hline $3-5$ & 270 & 22.4 & 1,080 & 22.4 & \\
\hline $6-11$ & 271 & 22.5 & 1,084 & 22.5 & \\
\hline $12-18$ & 223 & 18.5 & 892 & 18.5 & \\
\hline Mean (SD) & 6.18 & $(5.20)$ & 6.21 & $(5.17)$ & \\
\hline \multicolumn{6}{|l|}{ Gender } \\
\hline Girl & 596 & 49.5 & 2,384 & 49.5 & \\
\hline Boy & 607 & 50.5 & 2,428 & 50.5 & \\
\hline Urbanization & & & & & 0.56 \\
\hline 1 & 346 & 28.9 & 1,354 & 28.3 & \\
\hline 2 & 378 & 31.6 & 1,435 & 30.1 & \\
\hline 3 & 215 & 18.0 & 916 & 19.2 & \\
\hline 4 & 257 & 21.5 & 1,076 & 22.5 & \\
\hline Occupation & & & & & 0.03 \\
\hline White collar & 704 & 60.5 & 2,748 & 58.7 & \\
\hline Blue collar & 348 & 29.9 & 1,350 & 28.9 & \\
\hline Other & 112 & 9.62 & 582 & 12.4 & \\
\hline \multicolumn{6}{|l|}{ Allergic disease } \\
\hline Allergic conjunctivitis & 108 & 8.98 & 258 & 5.36 & $<0.0001$ \\
\hline Allergic rhinitis & 207 & 17.2 & 431 & 8.96 & $<0.0001$ \\
\hline Asthma & 104 & 8.65 & 252 & 5.24 & $<0.0001$ \\
\hline Atopic dermatitis & 84 & 6.98 & 176 & 3.66 & $<0.0001$ \\
\hline Urticaria & 50 & 4.16 & 116 & 2.41 & 0.0009 \\
\hline Number of allergic disease & & & & & $<0.0001$ \\
\hline 0 & 843 & 70.1 & 3,955 & 82.2 & \\
\hline 1 & 220 & 18.3 & 572 & 11.9 & \\
\hline 2 & 95 & 7.90 & 211 & 4.38 & \\
\hline$>2$ & 45 & 3.74 & 74 & 1.54 & \\
\hline$\chi^{2}$ test & & & & & \\
\hline
\end{tabular}

Table 4 presents the joint effect of ITP among ITP-associated allergic diseases. The top five risk factors of ITP (in descending order) were combinations of $\mathrm{AR}, \mathrm{AD}$, and urticaria $(\mathrm{OR}=5.88$; $95 \% \mathrm{CI}=1.57-22.0) ; \mathrm{AC}, \mathrm{AR}$, and urticaria $(\mathrm{OR}=4.90 ; 95 \%$ $\mathrm{CI}=1.41-17.0) ; \mathrm{AC}$ and urticaria $(\mathrm{OR}=4.20 ; 95 \% \mathrm{CI}=1.40-$ 12.6); $\mathrm{AC}, \mathrm{AR}$, and $\mathrm{AD}(\mathrm{OR}=4.03 ; 95 \% \mathrm{CI}=1.35-12.0)$; and $\mathrm{AR}$ and $\mathrm{AD}(\mathrm{OR}=3.61 ; 95 \% \mathrm{CI}=2.05-6.36)$, after accounting for age, sex, and parental occupation.

Table 5 shows the incidence rate and relative risk of allergic diseases in the children with ITP compared with the nonITP controls. The children with ITP consistently exhibited increased incidence rates and risks for all five allergic diseases, except urticaria.

\section{DISCUSSION}

Based on our review of the literature, this is the first large-scale study to evaluate the link between typical allergic diseases and ITP. The findings reveal that most of the allergic diseases investigated in this study, including $\mathrm{AR}, \mathrm{AD}$, urticaria, and $\mathrm{AC}$, were consistently associated with an increased risk of developing ITP both before and after the occurrence of allergic diseases. In addition, the risk of developing ITP increased with the number of concurrent allergic diseases, indicating that a higher burden of clinically evident allergic disease is associated with a higher prevalence of ITP.

AR presented the greatest risk of subsequent ITP development; the patients with AR had an $84 \%$ increased risk of subsequently developing ITP, and this observation was consistent among patients older than $3 \mathrm{y}$. The children with $\mathrm{AD}$ were at an increased risk of ITP, particularly those younger than $2 \mathrm{y}$, possibly because $\mathrm{AD}$ onset typically occurs in the first year of life, and is the first step in the allergic march (6-8); as children age, allergic diseases progress from $\mathrm{AD}$ to $\mathrm{AR}$, and asthma has classically been observed in some patients (6-8). However, in this study, we observed no association between ITP and asthma, potentially because the mean age of the onset of asthma among children in Taiwan (7.24 y) follows the mean age of the onset of ITP (6.18 y) (9).

Previous studies have proposed that an imbalance in Th1/ Th2 explains the development of Th2-mediated allergic diseases (3-5). However, the proposed Th1/Th2 paradigm appears unable to fully explain the increasing incidence of concurrent Th1-mediated autoimmune diseases. Recent studies have posited a link between allergies and autoimmune diseases (1012), and some studies have provided evidence of a relationship between allergies and ITP development. First, environmental factors such as viral or bacterial infection influence the development of allergic and autoimmune diseases (13). Second, previous studies have shown that FcyRIIb, a low-affinity IgG Fc receptor, plays a role in both allergic and autoimmune diseases, such as ITP. Intravenous immunoglobulin has been a standard treatment for ITP $(1,14)$; intravenous immunoglobulin functions by competitively inhibiting Fc-receptor binding sites and the activation of Fc $\gamma$ RIIb (15). Several studies have shown that intravenous immunoglobulin is also effective in treating refractory asthma, $\mathrm{AR}$, and $\mathrm{AD}(16-18)$. Fc $\gamma \mathrm{RIIb}$ has been plays a role in nasal eosinophilia in AR (19), and FcyRIIIa gene polymorphisms play a role in the pathogenesis of ITP and atopic disease $(20,21)$. Monoclonal antibodies against Fc $\gamma R I I I a$ have been shown to be effective in improving thrombocytopenia in ITP $(22,23)$. Third, elevated Th2 cytokines (IL-4, IL-10) have been observed in patients with ITP (24). Fourth, decreased regulatory $\mathrm{T}$ (Treg) cells exhibiting impaired function have been observed in ITP patients (25-31). The dysfunction of Treg cells plays a role in both autoimmune disease and allergic diseases (32-35). These studies have indicated an association between the pathogenesis of ITP and allergic disease, and thus support our finding that children with allergic diseases have a greater subsequent risk of developing ITP (and 
Table 2. The association between primary immune thrombocytopenia and single or multiple allergic diseases by sex

\begin{tabular}{lccc}
\hline Variable & All & Girl & Boy \\
\hline Allergic disease (yes vs. no) & & & \\
Allergic conjunctivitis & $1.39(1.09-1.79)^{* *}$ & $1.45(1.03-2.06)^{*}$ & $1.35(0.94-1.92)$ \\
Allergic rhinitis & $1.84(1.49-2.27)^{* * *}$ & $1.95(1.44-2.65)^{* * *}$ & $1.75(1.31-2.34)^{* * *}$ \\
Asthma & $1.05(0.80-1.38)$ & $1.02(0.67-1.54)$ & $1.09(0.76-1.57)$ \\
Atopic dermatitis & $1.66(1.25-2.21)^{* * *}$ & $1.88(1.21-2.92)^{* *}$ & $1.53(1.05-2.22)^{*}$ \\
Urticaria & $1.39(0.98-1.97)$ & $1.56(0.94-2.59)$ & $1.27(0.78-2.06)$ \\
Number of allergic disease ${ }^{b}$ & & 1.06 & 1.00 \\
0 & $1.94(1.54-2.19)^{* * *}$ & $1.77(1.38-2.27)^{* * *}$ & $1.91(1.49-2.44)^{* * *}$ \\
1 & $2.13(1.65-2.75)^{* * *}$ & $2.74(1.88-3.98)^{* * *}$ & $1.73(1.24-2.50)^{* *}$ \\
2 & $2.89(1.98-4.22)^{* * *}$ & $2.60(1.40-4.83)^{* *}$ & $3.09(1.91-4.99)^{* * *}$ \\
$>$ for trend & $<0.0001$ & $<0.0001$ & $<0.0001$ \\
\hline
\end{tabular}

a Manually adjusted for age, gender, parental occupation and comorbidity. ${ }^{\mathrm{C}}$ Manually adjusted for age, gender and occupation.

${ }^{*} P<0.05 ;{ }^{* *} P<0.01 ;{ }^{* * *} P<0.001$.

Table 3. The association between primary immune thrombocytopenia and allergic disease by age group

\begin{tabular}{|c|c|c|c|c|}
\hline Variable & $1-2 y$ & $3-5 y$ & $6-11 y$ & $12-18 y$ \\
\hline \multicolumn{5}{|l|}{ Allergic disease (yes vs. no) } \\
\hline Allergic conjunctivitis & $1.27(0.60-2.72)$ & $1.21(0.70-2.07)$ & $1.38(0.91-2.09)$ & $1.53(0.96-2.43)$ \\
\hline Asthma & $1.37(0.76-2.47)$ & $0.73(0.44-1.20)$ & $1.31(0.80-2.12)$ & $0.84(0.39-1.82)$ \\
\hline Atopic dermatitis & $1.62(1.09-2.41)^{*}$ & $1.45(0.84-2.51)$ & $4.02(1.76-9.19)^{* *}$ & $1.17(0.30-4.55)$ \\
\hline
\end{tabular}

Manually adjusted for gender, parental occupation, and comorbidity. ${ }^{*} P<0.05$; ${ }^{* *} P<0.01{ }^{* * *} P<0.001$.

Table 4. Joint effect of the association between primary immune thrombocytopenia (ITP) and allergic disease

\begin{tabular}{|c|c|c|c|c|c|c|}
\hline Allergic conjunctivitis & Allergic rhinitis & Atopic dermatitis & Urticaria & ITP no. & Non-ITP no. & OR $(95 \% \mathrm{Cl})$ \\
\hline- & - & - & - & 871 & 4,025 & 1.00 \\
\hline+ & - & - & - & 50 & 151 & $1.56(1.12-2.17)^{* *}$ \\
\hline- & - & + & - & 40 & 112 & $1.72(1.18-2.51)^{* * *}$ \\
\hline- & - & - & + & 21 & 68 & $1.44(0.88-2.36)$ \\
\hline+ & - & - & + & 6 & 7 & $4.20(1.40-12.6)^{*}$ \\
\hline- & + & + & - & 22 & 29 & $3.61(2.05-6.35)^{* * *}$ \\
\hline- & + & - & + & 8 & 17 & $2.22(0.96-5.18)$ \\
\hline- & - & + & + & 4 & 8 & $2.31(0.69-7.72)$ \\
\hline- & + & + & + & 5 & 4 & $5.88(1.57-22.0)^{* *}$ \\
\hline+ & + & + & + & 1 & 4 & $1.19(0.13-10.7)$ \\
\hline
\end{tabular}

Manually adjusted for age, gender and parental occupation.

${ }^{*} P<0.05$; ${ }^{*} P<0.01 ;{ }^{* *} P<0.001$. 
Table 5. Incidence and hazard ratio for different allergic disease compared to comparison

\begin{tabular}{|c|c|c|c|c|c|}
\hline \multirow[b]{2}{*}{ Allergic disease } & \multicolumn{2}{|c|}{$\begin{array}{c}\text { ITP } \\
N=843\end{array}$} & \multicolumn{2}{|c|}{$\begin{array}{l}\text { Non-ITP } \\
N=3,955\end{array}$} & \multirow{2}{*}{$\begin{array}{c}\text { Adjusted hazard } \\
\text { ratio }(95 \% \mathrm{Cl})\end{array}$} \\
\hline & Case & IR & Case & IR & \\
\hline Allergic conjunctivitis & 89 & 20.06 & 309 & 14.59 & $1.33(1.05-1.69)^{*}$ \\
\hline Allergic rhinitis & 171 & 42.52 & 350 & 16.62 & $2.48(2.06-2.98)^{* *}$ \\
\hline Asthma & 78 & 17.30 & 209 & 9.61 & $1.75(1.35-2.27)^{* *}$ \\
\hline Atopic dermatitis & 37 & 7.86 & 74 & 3.31 & $2.25(1.52-3.34)^{* * *}$ \\
\hline Urticaria & 31 & 6.52 & 101 & 4.54 & $1.43(0.96-2.14)$ \\
\hline
\end{tabular}

Manually adjusted for age, gender and parental occupation.

ITP, immune thrombocytopenia.

${ }^{*} P<0.05$; ${ }^{* *} P<0.001$.

vice versa). Thus, typical environmental triggers, genetic factors, and immunological aberrancies might contribute to the development of both disorders.

This study has a number of strengths. First, it robustly demonstrates the relationship between atopic diathesis and the risk of childhood ITP by using a high number of study patients. Second, we analyzed the effects not only of a specific type of allergic disease, but of numerous typical allergic diseases. Third, this study is a population-based study involving physician-diagnosed atopic diseases, which minimizes selection and recall bias. Fourth, we accounted for potentially confounding factors, including age, sex, and parental occupation.

Several limitations were encountered while conducting this study. This was a cross-sectional study, and the causality between allergic diseases and ITP could not be established. However, this study clearly demonstrates an association between allergic diseases and increased risk of ITP in a high number of patients. Another limitation was a lack of data on genetic and environmental factors that might have affected the risk of developing ITP and atopic diathesis. Ethnic influence was not considered in this study because most of Taiwan's population is of ethnic Chinese origin; thus, the results of this study might not be generalizable to other populations.

The findings of this study indicate that the onset of allergic diseases is associated with an increased risk of subsequent ITP. Furthermore, the risk of ITP was consistently associated with a number of comorbid allergic diseases. Future investigations on the environmental and genetic factors and common immunological aberrancies related to allergies and ITP are warranted.

\section{METHODS}

\section{Data Source}

The National Health Insurance Research Database (NHIRD), maintained by the National Health Research Institutes, is population-based data set derived from claims data from Taiwan's National Health Insurance program, a mandatory-enrollment single-payment system established in 1995, now covering more than $99 \%$ of Taiwan's population (36). This database contains all medical claims and information of insurants, thus providing a sufficient sample size to pursue the objectives of this study. To ensure the accuracy of disease diagnosis, Taiwan's National Health Insurance Bureau randomly reviews medical charts of one in every 100 ambulatory claims and one in every 20 inpatient claims. The high validity of the diagnostic data from the
NHIRD has been reported previously $(37,38)$. The children data set (age $<18$ y) was derived from the NHIRD and is also maintained by the National Health Research Institutes. The children data set contains $50 \%$ of children in the NHIRD, randomly selected from the children population from 1996 to 2008. Diseases are coded based on the International Classification of Diseases, Ninth Revision, Clinical Modification (ICD-9-CM). Because of personal electronic data privacy regulations, the insurants' records are encrypted before being released to researchers; thus, informed consent was not required in this study. Although the identification information was scrambled, this study also received approval from the Institutional Review Board at China Medical University Hospital (CMU-REC-101-012).

\section{Study Patients}

We enrolled children whose ITP was newly diagnosed between 1999 and 2008 as the ITP group. ITP was defined as ICD-9-CM Code 287.3, excluding Evan's syndrome (ICD-9-CM 287.32) and congenital and hereditary thrombocytopenic purpura (ICD-9-CM 287.33). To increase the likelihood of capturing a valid diagnosis of ITP, at least three claims coded in any diagnosis field as an inpatient or ambulatory claim for ITP within 12 mo were inclusion criteria for this study. The ITP diagnosis date was defined as the index date. Controls (the non-ITP group) were selected from the children without ITP before the entry date. Controls were frequency-matched to the case group according to age and sex at a ratio of 4:1.

\section{Definition of Variables and Comorbidities}

We grouped urbanization into seven levels based on research by Liu (38), where Level 1 was considered as the highest urbanization level. Because there were few children in Levels 5 to 7 , we combined these cases with Level 4. Comorbidities, including AC (ICD-9-CM 372.05, 372.10 and 372.14), AR (ICD-9-CM 477), asthma (ICD-9-CM 493), AD (ICD-9-CM 691.8), and urticaria (ICD-9-CM 708.0 and 708.9), were considered where at least one inpatient claim record or two ambulatory claims were present in any diagnosis field with respective ICD-9-CM codes. Furthermore, comorbidities were considered only in cases where diagnosis was made before the index date. The sum of allergic diseases was counted as the number of comorbidities.

\section{Statistical Analysis}

$\chi^{2}$ and $t$-tests were performed to test for differences in the baseline characteristics between the two groups. Unconditional logistic regression was used to estimate the ORs and 95\% CIs to evaluate the association between each allergic disease, the number of allergic diseases, and ITP. Multivariable logistic regression was used to manually account for the effects of age, sex, and comorbidities. We also estimated and compared the sex- and age-specific risk of developing ITP for both groups. For further analysis, we assessed the association between ITP and ITP-associated allergic diseases, including AC, AR, $\mathrm{AD}$, and urticaria. We also performed a cohort analysis on the incidence rate and relative risk of allergic diseases in children with ITP compared with the non-ITP controls to investigate a causative link between allergic diseases and the onset of ITP, or a common pathway between these disorders. This retrospective cohort study involved 843 patients (aged $<18$ y) whose ITP was newly diagnosed between 2000 and 2007 as the ITP cohort. The baseline was set as the date of ITP diagnosis. For each child with ITP, we randomly selected four nonITP children matched by sex, age (within 1-y intervals), urbanization of residential area, parental occupation, and baseline year. Children with pre-existing allergic diseases were excluded from both cohorts. Hazard ratios and $95 \%$ CIs were calculated using multivariable Cox proportional hazard regression models (with the non-ITP control cohort as the reference group) to assess the association between ITP and the risk of developing allergic diseases. SAS Version 9.3 (SAS Institute, Carey, NC) was used for the data analysis, and statistical significance was defined as a two-tailed $t$-test value of 0.05 .

\section{ACKNOWLEDGMENTS}

Ming-Ru Chiang and Chang-Ching Wei contributed equally to the conceptualization and design of the study, as well as drafting and approving the final version of the manuscript. Chin-Shin Muo, Lin-Shien Fu, and Tsai-Chung $\mathrm{Li}$ conducted the initial analysis, reviewed and revised the manuscript, and 
approved the final manuscript. Chia-Hung Kao coordinated and supervised the data collection process and critically reviewed the manuscript, and approved the final manuscript.

\section{STATEMENT OF FINANCIAL SUPPORT}

This study was partially supported by the Bureau of Health Promotion, Taiwan Ministry of Health Welfare, R.O.C. (Taiwan) (DOH99-HP-1205); study project grants (DMR-103-018 and DMR-103-020) from China Medical University Hospital, Taiwan Ministry of Health and Welfare Clinical Trial and Research Center of Excellence (MOHW103-TDU-B-212-113002); Health and welfare surcharge of tobacco products, China Medical University Hospital Cancer Research Center of Excellence (MOHW104-TD-B-111-03, Taiwan); and the International Research-Intensive Centers of Excellence, Taiwan (I-RiCE) (NSC101-2911-I-002-303). The funders had no role in the study design, data collection and analysis, decision to publish, or preparation of this manuscript. No additional external funding was received for this study.

Disclosure: The authors have no financial relationships relevant to this article to disclose.

\section{REFERENCES}

1. Lo E, Deane S. Diagnosis and classification of immune-mediated thrombocytopenia. Autoimmun Rev 2014;13:577-83.

2. Deane S, Teuber SS, Gershwin ME. The geoepidemiology of immune thrombocytopenic purpura. Autoimmun Rev 2010;9:A342-9.

3. Uzzaman A, Story R. Chapter 5: Allergic rhinitis. Allergy Asthma Proc 2012;33:Suppl 1:S15-8.

4. Sabin BR, Peters N, Peters AT. Chapter 20: Atopic dermatitis. Allergy Asthma Proc 2012;33:Suppl 1:S67-9.

5. Jutel M, Akdis CA. T-cell subset regulation in atopy. Curr Allergy Asthma Rep 2011;11:139-45.

6. Spergel JM, Paller AS. Atopic dermatitis and the atopic march. J Allergy Clin Immunol 2003;112(6 Suppl):S118-27.

7. Caimmi D, Marseglia A, Pieri G, Benzo S, Bosa L, Caimmi S. Nose and lungs: one way, one disease. Ital J Pediatr 2012;38:60.

8. Marrs T, Anagnostou K, Fitzsimons R, Fox AT. Optimising treatment of allergic rhinitis in children. Practitioner 2013;257:13-8, 2.

9. Strong C, Chang LY. Family socioeconomic status, household tobacco smoke, and asthma attack among children below 12 years of age: Gender differences. J Child Health Care 2014;18:388-98.

10. Rottem M, Gershwin ME, Shoenfeld Y. Allergic disease and autoimmune effectors pathways. Dev Immunol 2002;9:161-7.

11. Rottem M, Shoenfeld Y. Asthma as a paradigm for autoimmune disease. Int Arch Allergy Immunol 2003;132:210-4.

12. Shah $\mathrm{A}$. The pathologic and clinical intersection of atopic and autoimmune disease. Curr Allergy Asthma Rep 2012;12:520-9.

13. Kamradt T, Göggel R, Erb KJ. Induction, exacerbation and inhibition of allergic and autoimmune diseases by infection. Trends Immunol 2005;26:260-7.

14. Nugent DJ. IVIG in the treatment of children with acute and chronic idiopathic thrombocytopenic purpura and the autoimmune cytopenias. Clin Rev Allergy 1992;10:59-71.

15. Samuelsson A, Towers TL, Ravetch JV. Anti-inflammatory activity of IVIG mediated through the inhibitory Fc receptor. Science 2001;291:484-6.

16. Kimata $\mathrm{H}$. High dose gammaglobulin treatment for atopic dermatitis. Arch Dis Child 1994;70:335-6.

17. Rabinovitch N, Gelfand EW, Leung DY. The role of immunoglobulin therapy in allergic diseases. Allergy 1999;54:662-8.

18. Lamb SR, Rademaker M. Intravenous immunoglobulin therapy for the treatment of severe atopic dermatitis. Expert Opin Pharmacother 2001;2:67-74.

19. Watanabe T, Okano M, Hattori $\mathrm{H}$, et al. Roles of FcgammaRIIB in nasal eosinophilia and IgE production in murine allergic rhinitis. Am J Respir Crit Care Med 2004;169:105-12.
20. Fujimoto TT, Inoue M, Shimomura T, Fujimura K. Involvement of Fc gamma receptor polymorphism in the therapeutic response of idiopathic thrombocytopenic purpura. Br J Haematol 2001;115:125-30.

21. Zeyrek D, Tanac R, Altinoz S, et al. FcgammaRIIIa-V/F 158 polymorphism in Turkish children with asthma bronchiale and allergic rhinitis. Pediatr Allergy Immunol 2008;19:20-4.

22. Clarkson SB, Bussel JB, Kimberly RP, Valinsky JE, Nachman RL, Unkeless JC. Treatment of refractory immune thrombocytopenic purpura with an anti-Fc gamma-receptor antibody. N Engl J Med 1986;314:1236-9.

23. Soubrane C, Tourani JM, Andrieu JM, et al. Biologic response to antiCD16 monoclonal antibody therapy in a human immunodeficiency virusrelated immune thrombocytopenic purpura patient. Blood 1993;81:15-9.

24. Talaat RM, Elmaghraby AM, Barakat SS, El-Shahat M. Alterations in immune cell subsets and their cytokine secretion profile in childhood idiopathic thrombocytopenic purpura (ITP). Clin Exp Immunol 2014;176:291-300.

25. Aboul-Fotoh Lel-M, Abdel Raheem MM, El-Deen MA, Osman AM. Role of $\mathrm{CD} 4+\mathrm{CD} 25+\mathrm{T}$ cells in children with idiopathic thrombocytopenic purpura. J Pediatr Hematol Oncol 2011;33:81-5.

26. Catani L, Sollazzo D, Trabanelli S, et al. Decreased expression of indoleamine 2,3-dioxygenase 1 in dendritic cells contributes to impaired regulatory $\mathrm{T}$ cell development in immune thrombocytopenia. Ann Hematol 2013;92:67-78.

27. Sakakura M, Wada H, Tawara I, et al. Reduced $\mathrm{Cd} 4+\mathrm{Cd} 25+\mathrm{T}$ cells in patients with idiopathic thrombocytopenic purpura. Thromb Res 2007;120:187-93.

28. Liu B, Zhao H, Poon MC, et al. Abnormality of CD4(+)CD25(+) regulatory $\mathrm{T}$ cells in idiopathic thrombocytopenic purpura. Eur J Haematol 2007;78:139-43.

29. Ji L, Zhan Y, Hua F, et al. The ratio of Treg/Th17 cells correlates with the disease activity of primary immune thrombocytopenia. PLoS One 2012;7:e50909.

30. Zahran AM, Elsayh KI. CD4+ CD25+High Foxp3+ regulatory T cells, B lymphocytes, and Tlymphocytes in patients with acute ITP in Assiut Children Hospital. Clin Appl Thromb Hemost 2014;20:61-7.

31. Arandi N, Mirshafiey A, Jeddi-Tehrani M, et al. Alteration in frequency and function of $\mathrm{CD} 4^{+} \mathrm{CD} 25^{+} \mathrm{FOXP}^{+}$regulatory $\mathrm{T}$ cells in patients with immune thrombocytopenic purpura. Iran J Allergy Asthma Immunol 2014;13:85-92.

32. Bacchetta R, Gambineri E, Roncarolo MG. Role of regulatory T cells and FOXP3 in human diseases. J Allergy Clin Immunol 2007;120:227-35; quiz 236-7.

33. Kariyawasam HH, Rotiroti G. Allergic rhinitis, chronic rhinosinusitis and asthma: unravelling a complex relationship. Curr Opin Otolaryngol Head Neck Surg 2013;21:79-86

34. Robinson DS. The role of the T cell in asthma. J Allergy Clin Immunol 2010;126:1081-91; quiz 1092-3.

35. Lee JH, Yu HH, Wang LC, Yang YH, Lin YT, Chiang BL. The levels of $\mathrm{CD} 4+\mathrm{CD} 25+$ regulatory $\mathrm{T}$ cells in paediatric patients with allergic rhinitis and bronchial asthma. Clin Exp Immunol 2007;148:53-63.

36. Cheng TM. Taiwan's National Health Insurance system: high value for the dollar. In Okma KGH, Crivelli L, eds. Six Countries, Six Reform Models: The Health Reform Experience of Israel, the Netherlands, New Zealand, Singapore, Switzerland and Taiwan. Hackensack, NJ: World Scientific, 2009:71-204.

37. Cheng CL, Kao YH, Lin SJ, Lee CH, Lai ML. Validation of the National Health Insurance Research Database with ischemic stroke cases in Taiwan. Pharmacoepidemiol Drug Saf 2011;20:236-42.

38. Liu C, Hung Y, Chuang Y, et al. Incorporating development stratification of Taiwan townships into sampling design of large scale health interview survey. J Health Manage 2006;4:1-22.

39. Chang CC, Chang HC, Wu CH, Chang CY, Liao CC, Chen TL. Adverse postoperative outcomes in surgical patients with immune thrombocytopenia. Br J Surg 2013;100:684-92; discussion 693. 